\title{
Nonequilibrium dynamics in noncommutative spacetime
}

\author{
Chong-Sun $\mathrm{Chu}^{a}$ and Chiu Man $\mathrm{Ho}^{b, c, d}$ \\ ${ }^{a}$ Centre for Particle Theory and Department of Mathematics, Durham University, \\ Durham, DH1 3LE, U.K. \\ ${ }^{b}$ Department of Physics, University of California, \\ Berkeley, CA 94720, U.S.A. \\ ${ }^{c}$ Theoretical Physics Group, Lawrence Berkeley National Laboratory, \\ Berkeley, CA 94720, U.S.A. \\ ${ }^{d}$ Department of Physics and Astronomy, Vanderbilt University, \\ Nashville, Tennessee 37235, U.S.A. \\ E-mail: chong-sun.chu@durham.ac.uk, chiuman.ho@vanderbilt.edu
}

ABSTRACT: We study the effects of spacetime noncommutativity on the nonequilibrium dynamics of particles in a thermal bath. We show that the noncommutative thermal bath does not suffer from any further IR/UV mixing problem in the sense that all the finitetemperature non-planar quantities are free from infrared singularities. We also point out that the combined effect of finite temperature and noncommutative geometry has a distinct effect on the nonequilibrium dynamics of particles propagating in a thermal bath: depending on the momentum of the mode of concern, noncommutative geometry may switch on or switch off their decay and thermalization. This could have significant impacts on the nonequilibrium phenomena in the early universe at which spacetime noncommutativity may be present. Our results suggest a re-examination of some of the important processes in the early universe such as reheating after inflation, baryogenesis and the freeze-out of superheavy dark matter candidates.

KeYwords: Non-Commutative Geometry, Thermal Field Theory

ARXIV EPRINT: 0912.1748 


\section{Contents}

1 Introduction $\quad 1$

2 The model 3

2.1 Imaginary part of the self-energy 4

$\begin{array}{lll}2.2 & \text { Dispersion relation } & 7\end{array}$

3 Effects of noncommutativity on nonequilibrium dynamics 9

$\begin{array}{ll}3.1 & \text { Decay and thermalization rates } \\ & 10\end{array}$

3.2 Momentum dependent alternation of stability and thermalizability 11

4 Conclusions $\quad 14$

A Analysis of cases A and B 14

\section{Introduction}

On the noncommutative (NC) spacetime, the spacetime coordinates do not commute with each other anymore but obey the commutation relation:

$$
\left[x^{\mu}, x^{\nu}\right]=i \theta^{\mu \nu}
$$

where $\theta^{\mu \nu}$ is a constant antisymmetric matrix [1]. Noncommutative quantum field theory (NCQFT) can then be derived from its commutative counterpart with the usual product of fields replaced by the Moyal star product:

$$
\left.(\phi \star \chi)(x) \equiv e^{\frac{i}{2} \theta^{\mu \nu} \partial_{\mu}^{x} \partial_{\nu}^{y}} \phi(x) \chi(y)\right|_{y=x} .
$$

As NCQFT arises naturally from string theory [2-5], it has received lots of attentions and has been an active research topic during the last decade. NCQFT has many unique properties such as Lorentz violation [6], nonlocality and modified causality [7, 8]. Another intriguing phenomenon associated with NCQFT is the existence of the infrared (IR)/ultraviolet (UV) singularities [9-11]. This is a phenomenon which gives rise to various pathologies in the field theory. Despite the loss of Lorentz invariance and locality, it has been argued that both CPT and spin-statistics theorems still hold [12-14]. However, it has been pointed out that the space-time noncommutative theory (i.e. $\theta^{0 i} \neq 0$ for $i=1,2,3$ ) may violate unitarity [15] if the theory also suffers from IR/UV mixing $[16,17]$. Therefore, to avoid getting into trouble with unitarity, we will confine ourselves to the case with $\theta^{0 i}=0$ in our study.

On the other hand, nonequilibrium phenomena play a crucial role in many important processes in the early universe. These include reheating after inflation, baryogenesis, freezeout of dark matter candidates, electroweak and QCD phase transitions [18-20]. A common 
treatment of nonequilibrium evolution is to implement the closed-time-path (CTP) formalism [21-24] which is a path-integral approach to a time evolved density matrix. The thermal bath degrees of freedom are integrated out to obtain the nonequilibrium effective action which forms the generating functional for all the correlation functions. This approach also leads to quantum Boltzmann equations which can be solved to give the time evolution of the distribution functions. The accomplishment of thermal equilibrium is determined by the asymptotic time behaviour of the equal-time two-point correlation function and the distribution function.

A natural question to be asked would be: what if we consider finite temperature and spacetime noncommutativity at the same time? Since noncommutative geometry naturally introduces a new energy scale $E_{\mathrm{NC}} \sim \theta^{-1 / 2}$ in addition to the temperature scale $T$, noncommutativity could have an interesting impact on the time evolution of a nonequilibrium system. In particular, if spacetime noncommutativity is really present in the early universe, it would be important to understand how does it affect the relevant physics. Some behaviours of NCQFT at finite temperature have been investigated in [25-32]. However, none of these works have considered the decay and thermalization of particles propagating in a noncommutative thermal bath. In order to fill the gap, in this paper we raise and investigate the following question: how does spacetime noncommutativity affect the nonequilibrium dynamics?

In order to address this question, we consider a simple model with scalar $\Phi$ particles propagating in a thermal bath constituted by two other different scalars $\chi_{1}$ and $\chi_{2}$. The question of how do the $\Phi$ particles come from at the first place is irrelevant to our discussion. Immediately after the $\Phi$ particles are created, they are not in equilibrium with the thermal bath. They can either decay into $\chi_{1}+\chi_{2}$ or thermalize with them, or both, depending on their kinematical properties. A complete understanding of the nonequilibrium dynamics of $\Phi$ would be to study the time evolution of their correlation and distribution functions. However, as a first step, we will only confine ourselves to their decay and thermalization processes in this study. The decay rate $\Gamma_{\mathrm{D}}$ and thermalization rate $\Gamma_{\mathrm{T}}$ of the $\Phi$ particles are characterized by the imaginary part of their self-energy as well as their in-medium dispersion relation [33]. Notice that in the expanding early universe, even if $\Gamma_{\mathrm{T}} \neq 0$ and thereby a thermalization process is kinematically favored, it still does not guarantee an actual thermalization. To maintain thermal equilibrium with the thermal bath in the early universe, we require $\Gamma_{\mathrm{T}}>H$ where $H$ is the Hubble expansion rate. If $\Gamma_{\mathrm{T}}=0$ or $\Gamma_{\mathrm{T}}<H$, then the $\Phi$ particles will continue to be nonequilibrium; and whether they can have out-of-equilibrium decays depends on $\Gamma_{\mathrm{D}} \neq 0$ or $\Gamma_{\mathrm{D}}=0$.

This article is organized as follows. In section 2, we will outline our model and sketch how to compute the different contributions to the imaginary part of the self-energy, which will be analyzed to reveal its properties. We will also compute the real part of the selfenergy and hence obtain the noncommutative in-medium dispersion relation. The IR/UV mixing issue in our model will be analyzed as well. In section 3 , we will study the impacts of spacetime noncommutativity on the decay and thermalization processes of the $\Phi$ particles propagating in the thermal bath. We will find that as a combined result of finite temperature and noncommutative geometry, the stability and thermalizability properties 
of the $\Phi$ particles propagating in the thermal bath are altered in a momentum dependent manner. Finally, in section 4, we will give some preliminary discussions on the possible applications of our results to the early universe.

\section{The model}

We consider a theory of three interacting real scalar fields in noncommutative spacetime with the following Lagrangian density

$$
\mathcal{L}=\frac{1}{2} \partial_{\mu} \Phi \partial^{\mu} \Phi-\frac{1}{2} M_{B}^{2} \Phi^{2}+\sum_{i=1}^{2}\left[\frac{1}{2} \partial_{\mu} \chi_{i} \partial^{\mu} \chi_{i}-\frac{1}{2} M_{i}^{2} \chi_{i}^{2}\right]-\frac{g}{2} \chi_{1} \star \Phi \star \chi_{2}-\frac{g}{2} \chi_{2} \star \Phi \star \chi_{1}
$$

where $M_{B}$ is the bare mass of $\Phi$. We will assume that the mutual interaction between the fields $\chi_{1}, \chi_{2}$ ensures them to be in thermal equilibrium at a temperature $T=1 / \beta$. The commutative counterpart of this model has been previously studied in $[33,34]$ with an analysis of the different processes in the thermal medium. Here we will follow the similar treatment and conventions as [33].

The relevant quantity is the self-energy of the field $\Phi$ which we will obtain to one loop order $\mathcal{O}\left(g^{2}\right)$ in the Matsubara representation. The one-loop self-energy is given by

$$
\Sigma\left(\nu_{n}, \vec{k}\right)=-g^{2} \int \frac{d^{3} \vec{p}}{(2 \pi)^{3}} \frac{1}{\beta} \sum_{\omega_{m}} G_{\chi_{1}}\left(\omega_{m}, \vec{p}\right) G_{\chi_{2}}\left(\omega_{m}+\nu_{n}, \vec{p}+\vec{k}\right)\left(\frac{1}{2}+\frac{1}{2} e^{i p \times k}\right),
$$

where $\omega_{m}=2 \pi m / \beta, \nu_{n}=2 \pi n / \beta$ are the bosonic Matsubara frequencies and $p \times k \equiv$ $p_{i} \theta^{i j} k_{j}$. The factors $\frac{1}{2}$ and $\frac{1}{2} e^{i p \times k}$ represent the planar and non-planar contributions respectively. Obviously the noncommutative phase factor is nontrivial only if $k^{i}$ is nonvanishing in the direction where $\theta^{i j}$ is non-zero . The Matsubara propagators $G_{\chi_{1}}$ and $G_{\chi_{2}}$ are written in the following dispersive form

$$
\begin{aligned}
G_{\chi_{1}}\left(\omega_{m}, \vec{p}\right) & =\int d p_{0} \frac{\rho_{1}\left(p_{0}, \vec{p}\right)}{p_{0}-i \omega_{m}}, \\
G_{\chi_{2}}\left(\omega_{m}+\nu_{n}, \vec{p}+\vec{k}\right) & =\int d q_{0} \frac{\rho_{2}\left(q_{0}, \vec{p}+\vec{k}\right)}{q_{0}-i \omega_{m}-i \nu_{n}},
\end{aligned}
$$

where the spectral densities for $\chi_{1}$ and $\chi_{2}$ are

$$
\begin{aligned}
& \rho_{1}\left(p_{0}, \vec{p}\right)=\frac{1}{2 \omega_{1}}\left[\delta\left(p_{0}-\omega_{1}\right)-\delta\left(p_{0}+\omega_{1}\right)\right], \quad \omega_{1}=\sqrt{\vec{p}^{2}+M_{1}^{2}}, \\
& \rho_{2}\left(q_{0}, \vec{p}+\vec{k}\right)=\frac{1}{2 \omega_{2}}\left[\delta\left(q_{0}-\omega_{2}\right)-\delta\left(q_{0}+\omega_{2}\right)\right], \quad \omega_{2}=\sqrt{(\vec{p}+\vec{k})^{2}+M_{2}^{2}} .
\end{aligned}
$$

This representation allows us to carry out the sum over Matsubara frequencies $\omega_{m}$ in a rather straightforward manner $[35,36]$. The resulting self-energy can be further written in the dispersive form

$$
\Sigma\left(\nu_{n}, \vec{k}\right)=-\frac{1}{\pi} \int_{-\infty}^{\infty} d \omega \frac{\operatorname{Im} \Sigma_{\mathrm{R}}(\omega, k)}{\omega-i \nu_{n}}
$$

where $\operatorname{Im} \Sigma_{\mathrm{R}}(\omega, k)$ is the imaginary part of the retarded self-energy which is defined by the analytic continuation

$$
\Sigma_{\mathrm{R}}\left(k_{0}, k\right)=\Sigma\left(\nu_{n}=-i k_{0}-\epsilon, k\right) .
$$



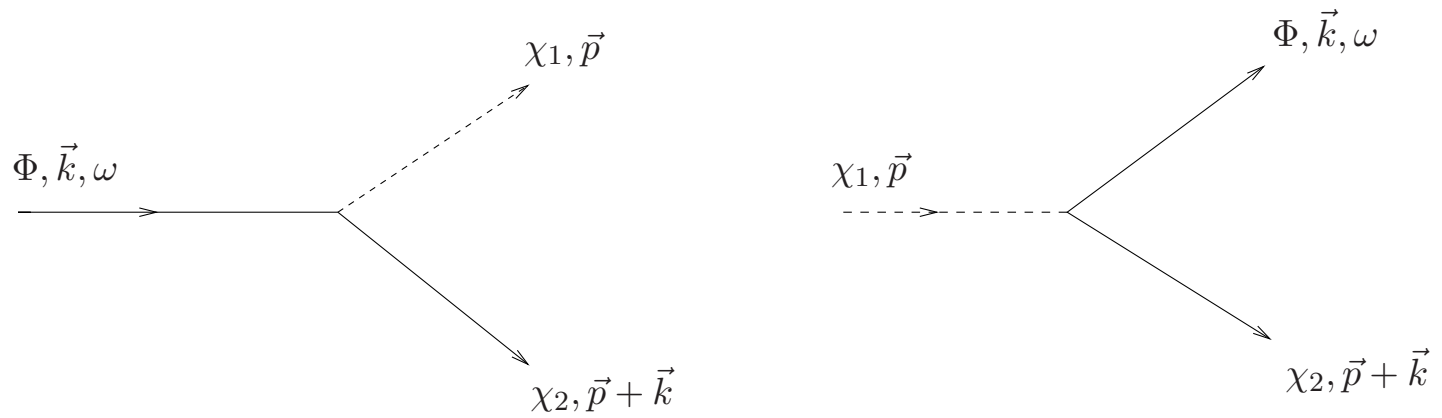

Figure 1. One-loop processes contributing to $\sigma_{0}^{\mathrm{P}, \mathrm{NP}}(\omega, k), \sigma_{a}^{\mathrm{P}, \mathrm{NP}}(\omega, k, T)$ and $\sigma_{b}^{\mathrm{P}, \mathrm{NP}}(\omega, k, T)$. The inverse processes are not shown.

\subsection{Imaginary part of the self-energy}

The retarded self-energy $\Sigma_{R}(\omega)$ has cuts along the real axis. The discontinuity across these cuts (defined by $\left.\Sigma_{\mathrm{R}}(\omega+i \epsilon)-\Sigma_{\mathrm{R}}(\omega-i \epsilon)\right)$ gives the imaginary part of $\Sigma_{\mathrm{R}}(\omega)$ :

$$
\operatorname{Disc} \Sigma_{\mathrm{R}}(\omega)=-2 i \operatorname{Im} \Sigma_{\mathrm{R}}(\omega) \text {. }
$$

It is then easy to obtain

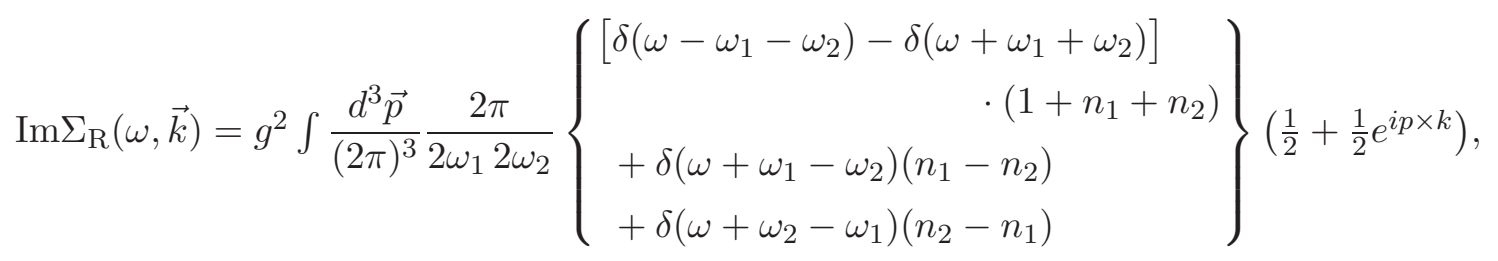

where

$$
n_{i}=n\left(\omega_{i}\right), \quad n(\omega)=\frac{1}{e^{\omega / T}-1} .
$$

Here the factor $\frac{1}{2}$ gives the planar contribution $\operatorname{Im} \Sigma_{\mathrm{R}}^{\mathrm{P}}$, while the factor $\frac{1}{2} e^{p \times k}$ gives the nonplanar contribution $\operatorname{Im} \Sigma_{\mathrm{R}}^{\mathrm{NP}}$. We will use the superscripts "P" and "NP" denote the planar and non-planar contributions respectively.

It is convenient to write $\operatorname{Im} \Sigma_{\mathrm{R}}^{\mathrm{P}, \mathrm{NP}}$ as a sum of several contributions of different physical origin, namely

$$
\begin{aligned}
\operatorname{Im} \Sigma_{\mathrm{R}}^{\mathrm{P}}(\omega, k, T) & =\sigma_{0}^{\mathrm{P}}(\omega, k)+\sigma_{a}^{\mathrm{P}}(\omega, k, T)+\sigma_{b}^{\mathrm{P}}(\omega, k, T), \\
\operatorname{Im} \Sigma_{\mathrm{R}}^{\mathrm{NP}}(\omega, k, T) & =\sigma_{0}^{\mathrm{NP}}(\omega, k)+\sigma_{a}^{\mathrm{NP}}(\omega, k, T)+\sigma_{b}^{\mathrm{NP}}(\omega, k, T) .
\end{aligned}
$$

Here the quantities $\sigma_{0}^{\mathrm{P}, \mathrm{NP}}(\omega, k)$ are the zero-temperature contributions, while $\sigma_{a}^{\mathrm{P}, \mathrm{NP}}(\omega, k, T), \sigma_{b}^{\mathrm{P}, \mathrm{NP}}(\omega, k, T)$ are the finite-temperature contributions. At one-loop, the processes that contribute to $\sigma_{0}^{\mathrm{P}, \mathrm{NP}}(\omega, k), \sigma_{a}^{\mathrm{P}, \mathrm{NP}}(\omega, k, T)$ are $\Phi \leftrightarrow \chi_{1}+\chi_{2}$, while the processes that contribute to $\sigma_{b}^{\mathrm{P}, \mathrm{NP}}(\omega, k, T)$ are $\chi_{1,2} \leftrightarrow \Phi+\chi_{2,1}$. See figure 1 .

In [33], the commutative version of our current model has been studied and the corresponding contributions to the imaginary part of the self-energy are computed as $\sigma_{0}, \sigma_{a}$ 
and $\sigma_{b}$ respectively. In fact, $\operatorname{Im} \Sigma_{\mathrm{R}}^{\mathrm{P}}$ is precisely $\frac{1}{2}$ times the corresponding results in [33], which implies that $\sigma_{0}^{\mathrm{P}}(\omega, k)=\frac{1}{2} \sigma_{0}, \sigma_{a}^{\mathrm{P}}(\omega, k, T)=\frac{1}{2} \sigma_{a}, \sigma_{b}^{\mathrm{P}}(\omega, k, T)=\frac{1}{2} \sigma_{b}$. We have:

$$
\begin{aligned}
\sigma_{0}^{\mathrm{P}}(\omega, k) & =\frac{g^{2}}{32 \pi k} \operatorname{sign}(\omega) \Theta\left[Q^{2}-\left(M_{1}+M_{2}\right)^{2}\right](B-A), \\
\sigma_{a}^{\mathrm{P}}(\omega, k, T) & =\frac{g^{2}}{32 \pi \beta k} \operatorname{sign}(\omega) \Theta\left[Q^{2}-\left(M_{1}+M_{2}\right)^{2}\right]\left[\ln \left(\frac{1-e^{-\beta B}}{1-e^{-\beta A}}\right)+\left(M_{1} \leftrightarrow M_{2}\right)\right], \\
\sigma_{b}^{\mathrm{P}}(\omega, k, T) & =\frac{g^{2}}{32 \pi \beta k} \operatorname{sign}(\omega) \Theta\left[\left(M_{1}-M_{2}\right)^{2}-Q^{2}\right]\left[\ln \left(\frac{1-e^{-\beta A}}{1-e^{-\beta B}}\right)+\left(M_{1} \leftrightarrow M_{2}\right)\right],
\end{aligned}
$$

where $A=\left|\omega_{p}^{-}\right|, B=\left|\omega_{p}^{+}\right|$,

$$
Q^{2}=\omega^{2}-k^{2}
$$

and

$$
\omega_{p}^{ \pm}=\frac{|\omega|\left[Q^{2}+\left(M_{1}^{2}-M_{2}^{2}\right)\right] \pm k \sqrt{\left[Q^{2}+\left(M_{1}^{2}-M_{2}^{2}\right)\right]^{2}-4 Q^{2} M_{1}^{2}}}{2 Q^{2}}
$$

In $\sigma_{a}^{\mathrm{P}}$ and $\sigma_{b}^{\mathrm{P}}, \beta k \ll 1$ corresponds to the high temperature limit while $\beta k \gg 1$ corresponds to the low temperature limit. Both $\sigma_{a}^{\mathrm{P}}$ and $\sigma_{b}^{\mathrm{P}}$ increases with decreasing $\beta k$. For instance, $\sigma_{a, b}^{\mathrm{P}} \rightarrow 0$ when $\beta k \rightarrow \infty$. On the other hand, both $\sigma_{a}^{\mathrm{P}}$ and $\sigma_{b}^{\mathrm{P}}$ approach to a finite value when $\beta k \rightarrow 0$.

As for the non-planar parts, we have to include the factor $e^{i p \times k}$ in the integral. To evaluate the integral, we note that since the vector $\theta^{i j} k_{j}$ is perpendicular to $k^{i}$, it is convenient to adopt a spherical coordinate system with the polar axis pointing in the $k^{i}$ direction and the azimuthal angle $\phi$ measured from an axis defined by $\theta^{i j} k_{j}$. Denoting the polar angle by $\vartheta$, we obtain

$$
p \times k=p \theta k \sin \vartheta \cos \phi,
$$

where $\theta$ is the magnitude of the vector $\theta^{i j} \hat{k}_{j}$ and $\hat{k}^{i}$ is the unit vector of $k^{i}$. For example, if $k^{i}=(0,0, k)$, then $\theta \equiv \sqrt{\left(\theta^{31}\right)^{2}+\left(\theta^{32}\right)^{2}}$. The calculations then proceed in a similar fashion as has been performed in the appendix of [33]. After lengthy but straightforward 
calculations, we obtain the non-planar contributions to the imaginary part of the self-energy

$$
\begin{aligned}
\sigma_{0}^{\mathrm{NP}}= & \frac{g^{2}}{16 \pi} \operatorname{sign}(\omega) \Theta\left[Q^{2}-\left(M_{1}+M_{2}\right)^{2}\right] \frac{1}{k Q \theta} \sin (\Omega k Q \theta), \\
\sigma_{a}^{\mathrm{NP}}= & \frac{g^{2}}{16 \pi} \operatorname{sign}(\omega) \Theta\left[Q^{2}-\left(M_{1}+M_{2}\right)^{2}\right] \sum_{n=1}^{\infty} \frac{e^{-\frac{B+A}{2 k} \beta k n}}{\beta k \sqrt{\left(\frac{Q \theta}{\beta}\right)^{2}-n^{2}}} \sin \left(\Omega \beta k \sqrt{\left(\frac{Q \theta}{\beta}\right)^{2}-n^{2}}\right) \\
& +\left(M_{1} \leftrightarrow M_{2}\right), \\
\sigma_{b}^{\mathrm{NP}}= & -\frac{g^{2}}{16 \pi} \operatorname{sign}(\omega)\left[\Theta\left(-Q^{2}\right) \sum_{n=1}^{\infty} \frac{e^{-\frac{B+A}{2 k} \beta k \sqrt{\left(\frac{Q \theta}{\beta}\right)^{2}+n^{2}}}}{\beta k \sqrt{\left(\frac{Q \theta}{\beta}\right)^{2}+n^{2}}} \sinh (\Omega \beta k n)\right. \\
& \left.+\Theta\left(Q^{2}\right) \Theta\left[\left(M_{1}-M_{2}\right)^{2}-Q^{2}\right] \sum_{n=1}^{\infty} \frac{e^{-\frac{B+A}{2 k} \beta k n}}{\beta k \sqrt{\left(\frac{Q \theta}{\beta}\right)^{2}-n^{2}}} \sin \left(\Omega \beta k \sqrt{\left(\frac{Q \theta}{\beta}\right)^{2}-n^{2}}\right)\right] \\
& +\left(M_{1} \leftrightarrow M_{2}\right),
\end{aligned}
$$

where $\Omega=(B-A) /(2 k)$ in the above expressions. ${ }^{1}$ We note that $\left|\sigma_{\mathrm{r}}^{\mathrm{NP}}\right| \leq\left|\sigma_{\mathrm{r}}^{\mathrm{P}}\right|$ for $\mathrm{r}=0, a, b$.

From the above expressions, it is obvious that $\sigma_{0}^{\mathrm{NP}} \rightarrow \sigma_{0}^{\mathrm{P}}$ when $k Q \theta \ll 1$. In fact, to the leading order, $Q \sim M_{0}$ where $M_{0}$ is the renormalized mass of $\Phi$ (to be determined in the next subsection). Thus, $k Q \theta \ll 1$ corresponds to the case where $\theta \ll\left(1 / M_{0}\right)(1 / k)$. Notice that $\sqrt{\theta} \equiv \lambda_{\mathrm{NC}}$ represents the characteristic length scale smaller than which the effect of noncommutative geometry becomes significant. Moreover, the Compton and deBroglie wavelengths associated with $\Phi$ can be identified as $\lambda_{\mathrm{C}} \sim 1 / M_{0}$ and $\lambda_{\mathrm{dB}} \sim 1 / k$ respectively. As a result, we conclude that $\sigma_{0}^{\mathrm{NP}} \rightarrow \sigma_{0}^{\mathrm{P}}$ when $\lambda_{\mathrm{NC}} \ll \sqrt{\lambda_{\mathrm{C}} \lambda_{\mathrm{dB}}}$. This is always true when $\lambda_{\mathrm{NC}}$ is much smaller than the smaller of $\lambda_{\mathrm{C}}$ and $\lambda_{\mathrm{dB}}$. In the relativistic case, $\lambda_{\mathrm{dB}} \ll \lambda_{\mathrm{C}}$; while in the non-relativistic case, $\lambda_{\mathrm{C}} \ll \lambda_{\mathrm{dB}}$. In any case, if $\lambda_{\mathrm{NC}}$ is really much smaller than the smaller of $\lambda_{\mathrm{C}}$ and $\lambda_{\mathrm{dB}}$, then the resolution due to either $\lambda_{\mathrm{C}}$ or $\lambda_{\mathrm{dB}}$ is not high enough to see the effect of noncommutativity. The system behaves as if it were commutative, and hence $\sigma_{0}^{\mathrm{NP}} \rightarrow \sigma_{0}^{\mathrm{P}}$. On the other hand, $\sigma_{0}^{\mathrm{NP}} \rightarrow 0$ when $k Q \theta \gg 1$, which corresponds to the case $\lambda_{\mathrm{NC}} \gg \sqrt{\lambda_{\mathrm{C}} \lambda_{\mathrm{dB}}}$. This is always true when $\lambda_{\mathrm{NC}}$ is larger than the larger of $\lambda_{\mathrm{C}}$ and $\lambda_{\mathrm{dB}}$. In this case, the system is completely noncommutative.

For $\sigma_{a}^{\mathrm{NP}}$ and $\sigma_{b}^{\mathrm{NP}}$, the significance of noncommutativite effect depends on the ratio $Q \theta / \beta \sim M_{0} T \theta$. When $Q \theta / \beta \ll 1$, noncommutativity is negligible. This corresponds to the case when $\lambda_{\mathrm{NC}} \ll \lambda_{\mathrm{T}}$ where $\lambda_{\mathrm{T}} \sim 1 / \sqrt{M_{0} T}$ can be identified as the thermal de-Broglie wavelength associated with $\Phi$ when it is propagating in the thermal bath with temperature $T$. In the thermal medium, it is the thermal de-Broglie wavelength that plays the role of the characteristic resolution acquired by $\Phi$. When $\lambda_{\mathrm{NC}} \ll \lambda_{\mathrm{T}}$, the system behaves as if it were commutative, and hence $\sigma_{a, b}^{\mathrm{NP}} \rightarrow \sigma_{a, b}^{\mathrm{P}}$. On the other hand, the effect of noncommutative

\footnotetext{
${ }^{1}$ Notice that if $(Q \theta / \beta)^{2}-n^{2}<0$, we replace $\sin (\ldots)$ by $\sinh (\ldots)$ and $\sqrt{(Q \theta / \beta)^{2}-n^{2}}$ by $\sqrt{n^{2}-(Q \theta / \beta)^{2}}$.
} 
geometry is significant when $Q \theta / \beta \gg 1$, which corresponds to $\lambda_{\mathrm{NC}} \gg \lambda_{\mathrm{T}}$. In this case, both $\sigma_{a}^{\mathrm{NP}}$ and $\sigma_{b}^{\mathrm{NP}}$ are suppressed.

In general, as $\theta$ increases, both $\sigma_{a}^{\mathrm{NP}}$ and $\sigma_{b}^{\mathrm{NP}}$ decrease. This can be attributed to the reduction of degrees of freedom due to spacetime noncommutativity. In particular, as $\theta \rightarrow \infty, \sigma_{a, b}^{\mathrm{NP}} \rightarrow 0$. In this case, the reduction of degrees of freedom due to spacetime noncommutativity is maximal. This can also be understood from the extremely rapid oscillations of the phase factor in the integrands of $\sigma_{a}^{\mathrm{NP}}$ and $\sigma_{b}^{\mathrm{NP}}$.

Furthermore, similar to the commutative case, $\beta k \ll 1$ corresponds to the high temperature limit while $\beta k \gg 1$ corresponds to the low temperature limit. This is true regardless of the relative significance of the noncommutative geometry. Both $\sigma_{a}^{\mathrm{NP}}$ and $\sigma_{b}^{\mathrm{NP}}$ increase with decreasing $\beta k$. For instance, $\sigma_{a, b}^{\mathrm{NP}} \rightarrow 0$ when $\beta k \rightarrow \infty$. On the other hand, both $\sigma_{a}^{\mathrm{NP}}$ and $\sigma_{b}^{\mathrm{NP}}$ approach to a finite value when $\beta k \rightarrow 0$.

\subsection{Dispersion relation}

The real part of the self-energy is given by

$$
\operatorname{Re} \Sigma_{\mathrm{R}}\left(\nu_{n}, \vec{k}\right)=-g^{2} \int \frac{d^{3} \vec{p}}{(2 \pi)^{3}} \frac{1}{\beta} \sum_{\omega_{m}} \frac{1}{\vec{p}^{2}+M_{1}^{2}+\omega_{m}^{2}} \frac{1}{(\vec{p}+\vec{k})^{2}+M_{2}^{2}+\left(\omega_{m}+\nu_{n}\right)^{2}}\left(\frac{1}{2}+\frac{1}{2} e^{i p \times k}\right) .
$$

Again, the factors $\frac{1}{2}$ and $\frac{1}{2} e^{i p \times k}$ represent the planar and non-planar contributions respectively. To facilitate the calculation, we introduce the Schwinger parameters

$$
\begin{aligned}
\frac{1}{\vec{p}^{2}+M_{1}^{2}+\omega_{m}^{2}} & =\int_{0}^{\infty} d \alpha_{1} e^{-\alpha_{1}\left(\vec{p}^{2}+M_{1}^{2}+\omega_{m}^{2}\right)}, \\
\frac{1}{(\vec{p}+\vec{k})^{2}+M_{2}^{2}+\left(\omega_{m}+\nu_{n}\right)^{2}} & =\int_{0}^{\infty} d \alpha_{2} e^{-\alpha_{2}\left[(\vec{p}+\vec{k})^{2}+M_{2}^{2}+\left(\omega_{m}+\nu_{n}\right)^{2}\right]} .
\end{aligned}
$$

By completing squares, the $p$ integrals now becomes Gaussian and can be readily evaluated to give

$$
\begin{aligned}
\operatorname{Re} \Sigma_{\mathrm{R}}^{\mathrm{P}, \mathrm{NP}}\left(\nu_{n}, \vec{k}\right)= & -\frac{g^{2}}{64 \pi^{2}} \int_{0}^{\infty} \frac{d \alpha}{\alpha} e^{-\alpha\left[\frac{1}{4} k_{E}^{2}+\frac{1}{2}\left(M_{1}^{2}+M_{2}^{2}\right)\right]-\frac{L^{2}}{\alpha}} \\
& \cdot \int_{-1}^{1} d x e^{-\frac{1}{4} \alpha k_{E}^{2} x^{2}-\frac{1}{2} \alpha\left(M_{1}^{2}-M_{2}^{2}\right) x} \vartheta\left(\frac{n}{2}(1-x), \frac{i \beta}{4 \pi \alpha}\right),
\end{aligned}
$$

where $\alpha=\alpha_{1}+\alpha_{2}, \quad x=\left(\alpha_{1}-\alpha_{2}\right) / \alpha, \quad k_{E}^{2}=k^{2}+\nu_{n}^{2}, \quad$ and

$$
\vartheta(z, \tau)=\sum_{m=-\infty}^{\infty} e^{2 \pi i m z+i \pi m^{2} \tau}
$$

is the Jacobi theta function [37]. Similar to [9], we have multiplied the above integrands by $\exp \left(-1 / \alpha \Lambda^{2}\right)$ in order to regulate the small $\alpha$ divergence such that

$$
L^{2}=\left\{\begin{array}{lll}
\frac{1}{\Lambda^{2}}, & \text { for } & \operatorname{Re} \Sigma_{\mathrm{R}}^{\mathrm{P}} \\
\frac{1}{\Lambda^{2}}+\frac{\tilde{k}^{2}}{4}, & \text { for } & \operatorname{Re} \Sigma_{\mathrm{R}}^{\mathrm{NP}}
\end{array},\right.
$$

where $\tilde{k}=k \theta$. 
The leading contribution of the integral (2.24) comes from the region $\alpha \sim 0$. After performing the above integrations and upon the analytic continuation $\nu_{n} \rightarrow-i \omega-\epsilon$, we obtain the retarded self-energy $\operatorname{Re} \Sigma_{\mathrm{R}}(\omega, k) \equiv I_{0}^{\mathrm{P}}+I_{0}^{\mathrm{NP}}+I^{\mathrm{P}}+I^{\mathrm{NP}}$ with

$$
\begin{aligned}
& I_{0}^{\mathrm{P}, \mathrm{NP}}=-\frac{g^{2}}{32 \pi^{2}} 2 K_{0}\left(2 c^{2} L^{2}\right) \\
& \approx \frac{g^{2}}{8 \pi^{2}} \ln (c L), \quad \text { for } c L \ll 1, \\
& I^{\mathrm{P}, \mathrm{NP}}=-\frac{g^{2} Q^{2}}{8 \pi^{2}(\beta \omega)^{2}} \sum_{m=1}^{\infty} \frac{1}{m^{2}}\left[\left(1+\frac{M_{1}^{2}-M_{2}^{2}}{Q^{2}}\right) \frac{L^{\prime}}{M_{1}} K_{1}\left(2 M_{1} L^{\prime}\right)\right. \\
& \left.+\left(1-\frac{M_{1}^{2}-M_{2}^{2}}{Q^{2}}\right) \frac{L^{\prime}}{M_{2}} K_{1}\left(2 M_{2} L^{\prime}\right)\right],
\end{aligned}
$$

where $Q^{2}=\omega^{2}-k^{2}=-k_{E}^{2} . K_{0}$ and $K_{1}$ are the modified Bessel functions of the second kind. Here

$$
c^{2}=\frac{\left(Q^{2}-\left(M_{1}-M_{2}\right)^{2}\right)\left(\left(M_{1}+M_{2}\right)^{2}-Q^{2}\right)}{4 Q^{2}}
$$

and

$$
L^{\prime 2}=L^{2}+\frac{m^{2} \beta^{2}}{4}
$$

In fact, $I_{0}^{\mathrm{P}, \mathrm{NP}}$ and $I^{\mathrm{P}, \mathrm{NP}}$ arise from the $m=0$ and $m \neq 0$ terms in the Jacobi theta function respectively. In the zero temperature limit $T \rightarrow 0$, the only non-vanishing term in the Jacobi theta function comes from $m=0$ in which case $\vartheta(z, \tau) \rightarrow 1$. Notice that $c$ is always positive-define if $\left(M_{1}-M_{2}\right)^{2}<Q^{2}<\left(M_{1}+M_{2}\right)^{2}$. For $Q^{2}>\left(M_{1}+M_{2}\right)^{2}$, the $\Phi$ particle can decay into $\chi_{1}$ and $\chi_{2}$. For $Q^{2}<\left(M_{1}-M_{2}\right)^{2}, \chi_{1}$ (or $\chi_{2}$ ) can decay into $\Phi$ and $\chi_{2}$ (or $\chi_{1}$ ). In both cases, $c$ becomes imaginary and a non-zero imaginary part will appear in $I_{0}^{\mathrm{P}, \mathrm{NP}}$.

The bare mass $M_{B}$ of $\Phi$ receives renormalization from both of $I_{0}^{\mathrm{P}}$ and $I_{0}^{\mathrm{NP}}$, and so the (zero-temperature) renormalized mass for $\Phi$ is defined as

$$
M_{0}^{2}=M_{B}^{2}+\left.I_{0}^{\mathrm{P}}\right|_{Q^{2}=M_{0}^{2}}+\left.I_{0}^{\mathrm{NP}}\right|_{Q^{2}=M_{0}^{2}}
$$

To the order $\mathcal{O}\left(g^{2}\right)$, the dispersion relation is then given by

$$
\omega^{2}=k^{2}+M_{0}^{2}+\mathcal{I}^{\mathrm{P}}+\mathcal{I}^{\mathrm{NP}}
$$

where

$$
\mathcal{I}^{\mathrm{P}}=\left.I^{\mathrm{P}}\right|_{Q^{2}=M_{0}^{2}} \quad \text { and } \quad \mathcal{I}^{\mathrm{NP}}=\left.I^{\mathrm{NP}}\right|_{Q^{2}=M_{0}^{2}}
$$

represent the finite-temperature corrections to the dispersion relation.

It is instructive to examine the behaviour of the finite temperature quantities $I^{\mathrm{P}}$, NP in various limits. In the low temperature limit with $T \ll M_{1}, M_{2}$, we have $M_{1,2} L^{\prime} \gg 1$. Using $K_{1}(x) \rightarrow \sqrt{\frac{\pi}{2 x}} e^{-x}$ for $x \gg 1$, it is obvious that $I^{\mathrm{P}, \mathrm{NP}}$ are exponentially suppressed as long as $T \ll M_{1}, M_{2}$, regardless of the significance of the noncommutativity. This is consistent with the fact that $I^{\mathrm{P}, \mathrm{NP}}$ arise from the $m \neq 0$ terms in the Jacobi theta function and can survive only at finite temperature. Similarly, $I^{\mathrm{NP}}$ is exponentially suppressed in 
the large $\theta$ limit with $1 / k \theta \ll M_{1}, M_{2}$, regardless of the magnitude of the temperature $T$. On the other hand, in the high temperature limit with $M_{1}, M_{2} \ll T, 1 / k \theta$, we have $M_{1,2} L^{\prime} \ll 1$. Using $K_{1}(x) \rightarrow \frac{1}{x}$ for $x \ll 1$, we obtain

$$
I^{\mathrm{P}, \mathrm{NP}} \approx-\frac{g^{2} Q^{2}}{96(\beta \omega)^{2}}\left[\left(1+\frac{M_{1}^{2}-M_{2}^{2}}{Q^{2}}\right) \frac{1}{M_{1}^{2}}+\left(1-\frac{M_{1}^{2}-M_{2}^{2}}{Q^{2}}\right) \frac{1}{M_{2}^{2}}\right] .
$$

It is remarkable to notice that the above expression for $I^{\mathrm{P}, \mathrm{NP}}$ are independent of $m$ and the ultraviolet cut-off $\Lambda$. Also, it is valid irrespective to the relative magnitude between $T$ and $1 / k \theta$. Most interestingly, the non-planar contribution $I^{\mathrm{NP}}$ is completely independent of $\theta$. This means that in the limit $M_{1}, M_{2} \ll T, 1 / k \theta$, the dispersion relation receives vanishing finite-temperature corrections from the spacetime noncommutativity.

Finally, let us comment on the IR/UV mixing effect of the noncommutative field theory at finite temperature. Needless to say, since $I_{0}^{\mathrm{NP}}$ is a zero-temperature non-planar quantity, it does suffer from the usual IR/UV mixing. Since the IR singularities are a reflection of the fact that the field theory is UV divergent, the key to resolve the IR singularities lies at a proper UV finite completion of the noncommutative field theory [38]. With a choice of UV completion, the IR singularities will get smoothen out. For example, a natural choice is to embed the noncommutative field theory as a low energy field theory of open string theory in background $B$-fields. It was shown explicitly in [38] that in doing so, the IR pathologies of noncommutative field theory are resolved. In particular, in the deep IR, the theory flows continuously to the commutative field theory and the normal Wilsonian behaviour is restored. Therefore, with this understanding, the zero-temperature IR/UV singularities are harmless.

It is clear that our noncommutative thermal bath does not suffer from any further IR/UV mixing problem, in the sense that all the finite-temperature non-planar quantities are healthy and are absent from any infrared singularities if we take the limit $k \rightarrow 0$ after taking the limit $\Lambda \rightarrow \infty$. For instance, let us look at the quantity $L^{\prime}$ in $I^{\mathrm{NP}}$ (which is of purely finite-temperature nature) as displayed in (2.29). It is obvious that $L^{\prime 2} \rightarrow m^{2} \beta^{2} / 4$ when $\Lambda \rightarrow \infty$ and $k \rightarrow 0$. Unless $T \rightarrow \infty, I^{\mathrm{NP}}$ is manifestly finite. Physically, it is the finite temperature $T$ acquired by the thermal bath that rescues the system from any further IR/UV mixing problem: the finite temperature $T$ acts as an effective ultraviolet cut-off for the system once we have taken the limit $\Lambda \rightarrow \infty$.

\section{Effects of noncommutativity on nonequilibrium dynamics}

In previous section, we have computed the imaginary part of the self-energy as well as the dispersion relation associated with the $\Phi$ particles. Now, we can proceed to study the nonequilibrium dynamics of $\Phi$ when it propagates in the noncommutative spacetime. In particular, we will focus on how does spacetime noncommutativity affect the possible decay and thermalization processes of $\Phi$ in the thermal bath. Without loss of generality, we will assume that $M_{1} \geq M_{2}$. 


\subsection{Decay and thermalization rates}

Let us write the imaginary part of the self-energy as $\operatorname{Im} \Sigma_{\mathrm{R}}=\sigma_{\mathrm{D}}+\sigma_{\mathrm{T}}$ where $\sigma_{\mathrm{D}}=$ $\sigma_{0}^{\mathrm{P}}+\sigma_{a}^{\mathrm{P}}+\sigma_{0}^{\mathrm{NP}}+\sigma_{a}^{\mathrm{NP}}$ and $\sigma_{\mathrm{T}}=\sigma_{b}^{\mathrm{P}}+\sigma_{b}^{\mathrm{NP}}$. These quantities are regulated by kinematical constraints and take the forms:

$$
\begin{aligned}
& \sigma_{\mathrm{D}}=\Theta\left[Q^{2}-\left(M_{1}+M_{2}\right)^{2}\right] \Lambda_{\mathrm{D}}\left(Q^{2}, k^{2}\right), \\
& \sigma_{\mathrm{T}}=\Theta\left[\left(M_{1}-M_{2}\right)^{2}-Q^{2}\right] \Lambda_{\mathrm{T}}\left(Q^{2}, k^{2}\right)+\Theta\left(-Q^{2}\right) \Lambda_{\mathrm{T}}^{\prime}\left(Q^{2}, k^{2}\right),
\end{aligned}
$$

where $\Lambda_{\mathrm{D}}\left(Q^{2}, k^{2}\right)$ can be read off from (2.13), (2.14), (2.19), (2.20), while $\Lambda_{\mathrm{T}}\left(Q^{2}, k^{2}\right)$ and $\Lambda_{\mathrm{T}}^{\prime}\left(Q^{2}, k^{2}\right)$ can be read off from $(2.15),(2.21)$ respectively. To compute the decay and thermalization rates $[33,36]$, we need to put the $\Phi$ field on-shell, i.e. setting

$$
Q^{2}=M_{0}^{2}+\mathcal{I}^{\mathrm{P}}+\mathcal{I}^{\mathrm{NP}}
$$

Then, to the order $\mathcal{O}\left(g^{2}\right)$, we obtain

$$
\Gamma_{\mathrm{D}}=\frac{\Lambda_{\mathrm{D}}\left(M_{0}^{2}, k^{2}\right)}{2 \omega_{0}} \Theta\left[Q^{2}-\left(M_{1}+M_{2}\right)^{2}\right]
$$

and

$$
\Gamma_{\mathrm{T}}=\frac{\Lambda_{\mathrm{T}}\left(M_{0}^{2}, k^{2}\right)}{2 \omega_{0}} \Theta\left[\left(M_{1}-M_{2}\right)^{2}-Q^{2}\right],
$$

where $\omega_{0}=\sqrt{k^{2}+M_{0}^{2}}$. Note that up to the order $\mathcal{O}\left(g^{2}\right)$, it is sufficient to set $Q^{2}=M_{0}^{2}$ in $\Lambda_{\mathrm{D}}$ and $\Lambda_{\mathrm{T}}$; however, one has to use the full expression (3.3) inside the $\Theta$ functions.

Physically, $\sigma_{0}^{\mathrm{P}}+\sigma_{0}^{\mathrm{NP}}$ represents the zero-temperature planar + non-planar contributions to the decay rate, while $\sigma_{a}^{\mathrm{P}}+\sigma_{a}^{\mathrm{NP}}$ represents the finite-temperature planar + nonplanar contributions to the decay rate. Similarly, $\sigma_{b}^{\mathrm{P}}+\sigma_{b}^{\mathrm{NP}}$ represents the finite-temperature planar + non-planar contributions to the thermalization rate. Notice that $\sigma_{b}^{\mathrm{P}}+\sigma_{b}^{\mathrm{NP}}$ is a purely finite-temperature effect and there is no zero-temperature counterpart of it, namely $\sigma_{b}^{\mathrm{P}}+\sigma_{b}^{\mathrm{NP}} \rightarrow 0$ as $T \rightarrow 0$.

The rates $\Gamma_{\mathrm{D}}(k)$ and $\Gamma_{\mathrm{T}}(k)$ depend on $k$, the magnitude of the spatial momentum. If $\Gamma_{\mathrm{D}}(k)=0$ for a certain $k$, then this mode of the $\Phi$ particle is stable in the thermal medium. Conversely, if $\Gamma_{\mathrm{D}}(k) \neq 0$ for a certain $k$, then this mode would be able to decay into $\chi_{1}+\chi_{2}$. Kinematically, the feasibility of decay is regulated by the Heaviside function

$$
\Theta\left[Q^{2}-\left(M_{1}+M_{2}\right)^{2}\right]
$$

On the other hand, $\Gamma_{\mathrm{T}}(k) \neq 0$ for a certain $k$ implies that this mode of $\Phi$ acquires a relaxation or thermalization time scale beyond which it approaches thermal equilibrium with the bath constituted by $\chi_{1}$ and $\chi_{2}$. This happens through the decay of $\chi_{1,2}$ into $\chi_{2,1}$ and $\Phi$, and their recombination, namely $\chi_{1,2} \leftrightarrow \chi_{2,1}+\Phi[33,36]$. As the modes carrying momentum number $k$ propagate through the thermal bath, they will be screened or dressed by the excitations in the medium and will propagate as quasi-particles. In fact, $\Gamma_{\mathrm{T}}(k)$ characterizes the "decay rate" of the quasi-particles associated with $\Phi$ in the medium, and this is precisely the relaxation or thermalization rate $[33,36]$. Of course, if $\Gamma_{\mathrm{T}}(k)=0$, 
then it takes an infinitely long time for these modes of $\Phi$ to approach thermal equilibrium with the bath, which simply means that these modes can never thermalize with the bath. Kinematically, the feasibility of thermalization is regulated by the Heaviside function

$$
\Theta\left[\left(M_{1}-M_{2}\right)^{2}-Q^{2}\right] .
$$

\subsection{Momentum dependent alternation of stability and thermalizability}

What we would like to study is the following. How does the conventional understanding of the dynamics of $\Phi$ in the thermal bath change in the presence of noncommutative geometry?

Since the decay and thermalization processes depend crucially on the relative size of $Q^{2}$ compared to $\left(M_{1}-M_{2}\right)^{2}$ and $\left(M_{1}+M_{2}\right)^{2}$, it is useful to consider the following three regions of $Q^{2}$ :

$$
\begin{aligned}
& \mathcal{R}_{1}: Q^{2}<\left(M_{1}-M_{2}\right)^{2}, \quad \text { where we have } \Gamma_{\mathrm{T}} \neq 0 \text { and } \Gamma_{\mathrm{D}}=0, \\
& \mathcal{R}_{2}:\left(M_{1}-M_{2}\right)^{2}<Q^{2}<\left(M_{1}+M_{2}\right)^{2}, \quad \text { where we have } \Gamma_{\mathrm{T}}=\Gamma_{\mathrm{D}}=0, \\
& \mathcal{R}_{3}:\left(M_{1}+M_{2}\right)^{2}<Q^{2}, \quad \text { where we have } \Gamma_{\mathrm{T}}=0 \text { and } \Gamma_{\mathrm{D}} \neq 0 .
\end{aligned}
$$

If we define $Q_{\mathrm{C}}^{2}$ as the commutative counterpart of $Q^{2}$, then it would be given by $Q_{\mathrm{C}}^{2}=$ $M_{0}^{2}+2 \mathcal{I}^{\mathrm{P}}$. When one turns on the noncommutativity parameter, $Q_{\mathrm{C}}^{2}$ changes to $Q^{2}$ and could cross over from one region to another, corresponding to turning on or turning off the decay and/or the thermalization processes as one takes into account of the effects of noncommutativity. What can actually happen depends on the "jump" $Q^{2}-Q_{\mathrm{C}}^{2}=\mathcal{I}^{\mathrm{NP}}-\mathcal{I}^{\mathrm{P}}$. Since $\left|\mathcal{I}^{\mathrm{NP}}\right| \leq\left|\mathcal{I}^{\mathrm{P}}\right|$, we will always have $Q^{2}-Q_{\mathrm{C}}^{2}<0$ if $\mathcal{I}^{\mathrm{P}}>0$ and $Q^{2}-Q_{\mathrm{C}}^{2}>0$ if $\mathcal{I}^{\mathrm{P}}<0$, regardless of the signs of $\mathcal{I}^{\mathrm{NP}}$. Therefore the direction of the "jump" depends solely on the sign of $\mathcal{I}^{\mathrm{P}}$. By setting $\Lambda \rightarrow \infty$ and $Q^{2}=M_{0}^{2}$ in (2.29), it is easy to obtain that

$$
\begin{array}{lll}
\mathcal{I}^{\mathrm{P}}>0 & \text { when } & M_{0}^{2}<\frac{\left(M_{1}^{2}-M_{2}^{2}\right)^{2}}{M_{1}^{2}+M_{2}^{2}}, \\
\mathcal{I}^{\mathrm{P}}<0 \quad \text { when } & M_{1}^{2}-M_{2}^{2}<M_{0}^{2}
\end{array}
$$

for any temperature $T$ and momentum $k$. And for $M_{0}^{2}$ in the intermediate region, one obtains

$$
\left.\begin{array}{ll}
\mathcal{I}^{\mathrm{P}}>0 & \text { for } T<T_{0}, \\
\mathcal{I}^{\mathrm{P}}<0 & \text { for } T>T_{0},
\end{array}\right\} \quad \text { when } \quad \frac{\left(M_{1}^{2}-M_{2}^{2}\right)^{2}}{M_{1}^{2}+M_{2}^{2}} \leq M_{0}^{2} \leq M_{1}^{2}-M_{2}^{2} .
$$

Here $T_{0}$ is a temperature that is determined by solving $\mathcal{I}^{\mathrm{P}}\left(T_{0}\right)=0$. Note that $T_{0}$ depends not just on the mass $M_{0}$ of the propagating particles $\Phi$ and the properties of the thermal bath (i.e. $M_{1}, M_{2}$ ), but also on the momentum $k$ of the mode of $\Phi$ in consideration. Therefore, as we remarked above, the impact of spacetime noncommutativity on the decay and thermalization processes is going to be momentum dependent. We remark that due to the rather complicated form for $\mathcal{I}^{\mathrm{P}}$, an analytic expression for $T_{0}$ is not available. Fortunately, this is actually not needed in our analysis below which is aimed at explaining the general physical features. In a concrete phenomenological study, a more detailed knowledge of $T_{0}$ maybe needed and this can always be obtained numerically. 
As we mentioned above, the decay and thermalization processes may be turned on or off when one takes into account of the effects of noncommutativity. This happens whenever $Q^{2}$ crosses over different regions as noncommutativity is turned on. As a result, we obtain the following six interesting cases which may happen in principle.

1. $Q_{C}^{2}$ in region $\mathcal{R}_{1}: \quad Q_{C}^{2}<\left(M_{1}-M_{2}\right)^{2}$

In the commutative picture, we have $\Gamma_{\mathrm{D}}=0$ and $\Gamma_{\mathrm{T}} \neq 0$ which imply that $\Phi$ is stable and cannot decay into $\chi_{1}+\chi_{2}$, but it can thermalize with the bath. Once we switch to the noncommutative bath, we may have modes such that

- Case A: $\left(M_{1}-M_{2}\right)^{2}<Q^{2}<\left(M_{1}+M_{2}\right)^{2} \Longrightarrow \Gamma_{\mathrm{D}}=\Gamma_{\mathrm{T}}=0$. This corresponds to the situation with $\mathcal{I}^{\mathrm{P}}<0$. These modes of $\Phi$ are still stable against decay into $\chi_{1}+\chi_{2}$, but they cannot thermalize with the noncommutative bath anymore. In this case, we see that noncommutative geometry suppresses thermalization for these modes.

- Case B: $\left(M_{1}+M_{2}\right)^{2}<Q^{2} \Longrightarrow \Gamma_{\mathrm{D}} \neq 0$ and $\Gamma_{\mathrm{T}}=0$. This also corresponds to the situation with $\mathcal{I}^{\mathrm{P}}<0$. These modes of $\Phi$ which are originally stable in the commutative bath, can now decay into $\chi_{1}+\chi_{2}$ in the noncommutative bath, but they cannot thermalize with it anymore. In this case, noncommutative geometry induces decay but suppresses thermalization for these modes.

2. $Q_{C}^{2}$ in region $\mathcal{R}_{2}: \quad\left(M_{1}-M_{2}\right)^{2}<Q_{C}^{2}<\left(M_{1}+M_{2}\right)^{2}$

In the commutative picture, we have $\Gamma_{\mathrm{D}}=\Gamma_{\mathrm{T}}=0$ which implies that $\Phi$ can neither decay into $\chi_{1}+\chi_{2}$ nor thermalize with the bath. Once we switch to the noncommutative bath, we may have modes such that

- Case C: $0<Q^{2}<\left(M_{1}-M_{2}\right)^{2} \Longrightarrow \Gamma_{\mathrm{D}}=0$ and $\Gamma_{\mathrm{T}} \neq 0$. This corresponds to the situation with $\mathcal{I}^{\mathrm{P}}>0$. These modes of $\Phi$ still cannot decay into $\chi_{1}+\chi_{2}$, but they can now thermalize with the noncommutative bath. In this case, noncommutative geometry induces thermalization for these modes.

- Case D: $\quad\left(M_{1}+M_{2}\right)^{2}<Q^{2} \Longrightarrow \Gamma_{\mathrm{D}} \neq 0$ and $\Gamma_{\mathrm{T}}=0$. This corresponds to the situation with $\mathcal{I}^{\mathrm{P}}<0$. These modes of $\Phi$ can now decay into $\chi_{1}+\chi_{2}$ in the noncommutative bath, but still cannot thermalize with it. In this case, noncommutative geometry induces decay for these modes.

3. $Q_{C}^{2}$ in region $\mathcal{R}_{3}: \quad\left(M_{1}+M_{2}\right)^{2}<Q_{\mathrm{C}}^{2}$

In the commutative picture, we have $\Gamma_{\mathrm{D}} \neq 0$ and $\Gamma_{\mathrm{T}}=0$ which imply $\Phi$ is unstable and can decay into $\chi_{1}+\chi_{2}$, but it cannot thermalize with the bath. Once we switch to the noncommutative bath, we may have modes such that

- Case E: $0<Q^{2}<\left(M_{1}-M_{2}\right)^{2} \Longrightarrow \Gamma_{\mathrm{D}}=0$ and $\Gamma_{\mathrm{T}} \neq 0$. This corresponds to the situation with $\mathcal{I}^{\mathrm{P}}>0$. These modes of $\Phi$ cannot decay into $\chi_{1}+\chi_{2}$ in the noncommutative bath anymore, but they can now thermalize with it. In this 
case, noncommutative geometry suppresses decay but induces thermalization for these modes.

- Case F: $\quad\left(M_{1}-M_{2}\right)^{2}<Q^{2}<\left(M_{1}+M_{2}\right)^{2} \Longrightarrow \Gamma_{\mathrm{D}}=\Gamma_{\mathrm{T}}=0$. This also corresponds to the situation with $\mathcal{I}^{\mathrm{P}}>0$. These modes of $\Phi$ cannot decay into $\chi_{1}+\chi_{2}$ in the noncommutative bath anymore, and they still cannot thermalize with it. In this case, noncommutative geometry suppresses decay for these modes.

For a given thermal bath and a propagating particle, the set of masses $M_{0}, M_{1}, M_{2}$ and the coupling $g$ are fixed. To decide which of the above six cases can occur, one need to examine carefully how $Q^{2}$ changes when noncommutativity is turned on. As an illustration, we present the detailed analysis of cases $\mathrm{A}$ and $\mathrm{B}$ in the appendix $\mathrm{A}$. The result is that cases A and B can occur only if the masses of the thermal bath satisfy

$$
\frac{M_{1}}{M_{2}}>4+\sqrt{15}
$$

and if $M_{0}$ falls in the range of

$$
\frac{\left(M_{1}^{2}-M_{2}^{2}\right)^{2}}{M_{1}^{2}+M_{2}^{2}}<M_{0}^{2}<\frac{5}{4}\left(M_{1}-M_{2}\right)^{2} .
$$

When these conditions are satisfied, then depending on the momentum $k$ of the mode of concern, either case A or case B or both cases can occur. See (A.17)-(A.23).

Similar analysis can be performed for the other four cases. The conclusion is similar. For cases $\mathrm{E}$ and $\mathrm{F}$ to occur, the necessary conditions are that the masses of thermal bath has to satisfy

$$
M_{1} / M_{2}>11
$$

and $M_{0}$ has be in the range

$$
\frac{5}{6}\left(M_{1}+M_{2}\right)^{2}<M_{0}^{2}<M_{1}^{2}-M_{2}^{2} .
$$

For cases $\mathrm{C}$ and $\mathrm{D}$, no condition is needed on $M_{1} / M_{2}$. But (3.15) is needed for the case C and (A.6) is needed for the case D to occur. Similar conditions as (A.17)-(A.23) can be written down for these four cases. We will skip them here.

All in all, we conclude that all of the six cases A-F listed above can occur in general. Depending on the masses, temperature of the thermal bath and the magnitude $k$ of the spatial momentum of the mode of concern, the decay and thermalization rates of $\Phi$ can be either induced or suppressed when one takes into account of noncommutativity properly. In general, there is a window of momentum modes whose stability and/or thermalizability properties are altered. The window depends on the temperature of the thermal bath. Typically, the affected modes are in the high (respectively low) $k$ regime when $T$ is low (respectively high). 


\section{Conclusions}

In this article, we studied the nonequilibrium dynamics of a scalar $\Phi$ propagating through a noncommutative thermal bath. We showed that noncommutative geometry has a distinct impact on the nonequilibrium dynamics of particles propagating in a thermal bath by providing a momentum dependent enhancement or suppression of their decay or thermalization processes. This is a combined effect of finite temperature and noncommutative geometry. Also, we pointed out that the finite temperature $T$ of the thermal bath can play the role as an effective ultraviolet cut-off which rescues all the finite-temperature non-planar quantities from any further IR/UV mixing problem.

Although our thermal bath is represented in a specific way by the two scalar particles $\chi_{1}$ and $\chi_{2}$, our analysis is general and our conclusions should apply generally for any bosons in contact with a noncommutative thermal bath.

A particularly interesting arena of application of our results is the early universe at which spacetime noncommutativity may be present. Our results call for a re-examination of some of the important processes in the early universe such as reheating after inflation, baryogenesis and the freeze-out of superheavy dark matter candidates, which are generally believed to occur at very high energy scales where spacetime noncommutativity could be significant enough. It will be very interesting to re-examine these processes in details and see how the presence of noncommutative geometry could affect the conventional picture. This could also provide another channel for probing the presence of noncommutative geometry in the early universe, in addition to the possible cosmological imprint left by inflation, see for example [39-41].

\section{Acknowledgments}

C. S. Chu would like to thank the Berkeley Center for Theoretical Physics for hospitality during his visit. C. M. Ho would like to thank Ori Ganor for useful discussions. The work of C. S. Chu has been supported by EPSRC and STFC.

\section{A Analysis of cases A and B}

Modes for which the cases A and B can occur have to satisfy the following conditions:

$$
\begin{aligned}
M_{0}^{2}+2 \mathcal{I}^{\mathrm{P}} & <\left(M_{1}-M_{2}\right)^{2}, \\
\left|\mathcal{I}^{\mathrm{P}}\right| & <0.1 M_{0}^{2}, \\
\left(M_{1}-M_{2}\right)^{2}<M_{0}^{2}+\mathcal{I}^{\mathrm{P}}+\mathcal{I}^{\mathrm{NP}} & <\left(M_{1}+M_{2}\right)^{2}, \\
\left(M_{1}+M_{2}\right)^{2}<M_{0}^{2}+\mathcal{I}^{\mathrm{P}}+\mathcal{I}^{\mathrm{NP}} &
\end{aligned}
$$

Here the first condition specifies that one is initially in the region $\mathcal{R}_{1}$. The second condition (A.2) is a condition on the size of the thermal correction to $M_{0}^{2}$. We have imposed a conservative $10 \%$ correction so as to guarantee that we are in the validity regime of the perturbation theory. In principle, one could have taken a different value for the RHS of (A.2), 
e.g. $0.2 M_{0}^{2}$. This will not modify the analysis below and the physical effects we are going to point out are generic. The condition (A.3) is for case A and the condition (A.4) is for case B.

To analyze these conditions, we first note that cases $\mathrm{A}$ and $\mathrm{B}$ can occur only if $\mathcal{I}^{\mathrm{P}}<0$. This means that it is necessary to require $M_{0}^{2}>\left(M_{1}^{2}-M_{2}^{2}\right)^{2} /\left(M_{1}^{2}+M_{2}^{2}\right)$. On the other hand, the conditions (A.1) and (A.2) imply that

$$
\frac{1}{2}\left[M_{0}^{2}-\left(M_{1}-M_{2}\right)^{2}\right]<-\mathcal{I}^{\mathrm{P}}<0.1 M_{0}^{2} .
$$

This is a non-empty condition for $\mathcal{I}^{\mathrm{P}}$ only if $M_{0}^{2}<\frac{5}{4}\left(M_{1}-M_{2}\right)^{2}$. This condition, together with the previous condition on $M_{0}^{2}$, gives

$$
\frac{\left(M_{1}^{2}-M_{2}^{2}\right)^{2}}{M_{1}^{2}+M_{2}^{2}}<M_{0}^{2}<\frac{5}{4}\left(M_{1}-M_{2}\right)^{2} .
$$

This specifies a non-empty range for $M_{0}^{2}$ only if $\left(M_{1}^{2}-M_{2}^{2}\right)^{2} /\left(M_{1}^{2}+M_{2}^{2}\right)<\frac{5}{4}\left(M_{1}-M_{2}\right)^{2}$, i.e.

$$
\frac{M_{1}}{M_{2}}>4+\sqrt{15}
$$

The condition (A.7) on the ratio $M_{1} / M_{2}$ and the condition (A.6) on $M_{0}$ are common to cases $\mathrm{A}$ and $\mathrm{B}$ and are the necessary conditions for the cases $\mathrm{A}$ and $\mathrm{B}$ to occur. Now assume (A.7) and (A.6) are satisfied, we are guaranteed that (A.5) specifies a non-empty range for $-\mathcal{I}^{\mathrm{P}}$. The question is whether and for what configuration of modes will (A.5) be satisfied. To address this, we need the form of $\mathcal{I}^{\mathrm{P}}$ as a function of $k$ and $T$, which we collect here (also for $\mathcal{I}^{\mathrm{NP}}$ ):

$$
\begin{aligned}
\mathcal{I}^{\mathrm{P}} & =-\frac{g^{2} M_{0}^{2}}{16 \pi^{2} \omega^{2}} T^{2} \sum_{m=1}^{\infty}\left[\frac{c_{+}}{m^{2} M_{1}^{2}} h\left(m x_{1}\right)+\frac{c_{-}}{m^{2} M_{2}^{2}} h\left(m x_{2}\right)\right] \\
\mathcal{I}^{\mathrm{NP}} & =-\frac{g^{2} M_{0}^{2}}{16 \pi^{2} \omega^{2}} T^{2} \sum_{m=1}^{\infty}\left[\frac{c_{+}}{m^{2} M_{1}^{2}} h\left(m y_{1}\right)+\frac{c_{-}}{m^{2} M_{2}^{2}} h\left(m y_{2}\right)\right] .
\end{aligned}
$$

Here $c_{ \pm}=1 \pm\left(M_{1}^{2}-M_{2}^{2}\right) / M_{0}^{2}$

$$
x_{i}=M_{i} / T, \quad y_{i}=\sqrt{1 / T^{2}+k^{2} \theta^{2}} M_{i}, \quad i=1,2
$$

and

$$
h(x):=x K_{1}(x) .
$$

To analyze which set of modes satisfy the condition (A.5), we note that $-\mathcal{I}^{\mathrm{P}}=0$ at $T=0$ and $-\mathcal{I}^{\mathrm{P}} \sim d \times T^{2}$ for large $T \gg M_{1}, M_{2}$, where $d \approx g^{2} M_{0}^{2} /\left(96 \omega^{2}\right)\left[c_{+} / M_{1}^{2}+c_{-} / M_{2}^{2}\right]>0$. Therefore, for any given $k$, there is a temperature range

$$
T_{1}(k)<T<T_{2}(k)
$$

such that (A.5) is satisfied. Here the temperatures $T_{1,2}(k)$ are determined by solving

$$
\mathcal{I}^{\mathrm{P}}\left(k, T_{1}(k)\right)=\frac{1}{2}\left[M_{0}^{2}-\left(M_{1}-M_{2}\right)^{2}\right] \quad \text { and } \quad \mathcal{I}^{\mathrm{P}}\left(k, T_{2}(k)\right)=0.1 M_{0}^{2} .
$$




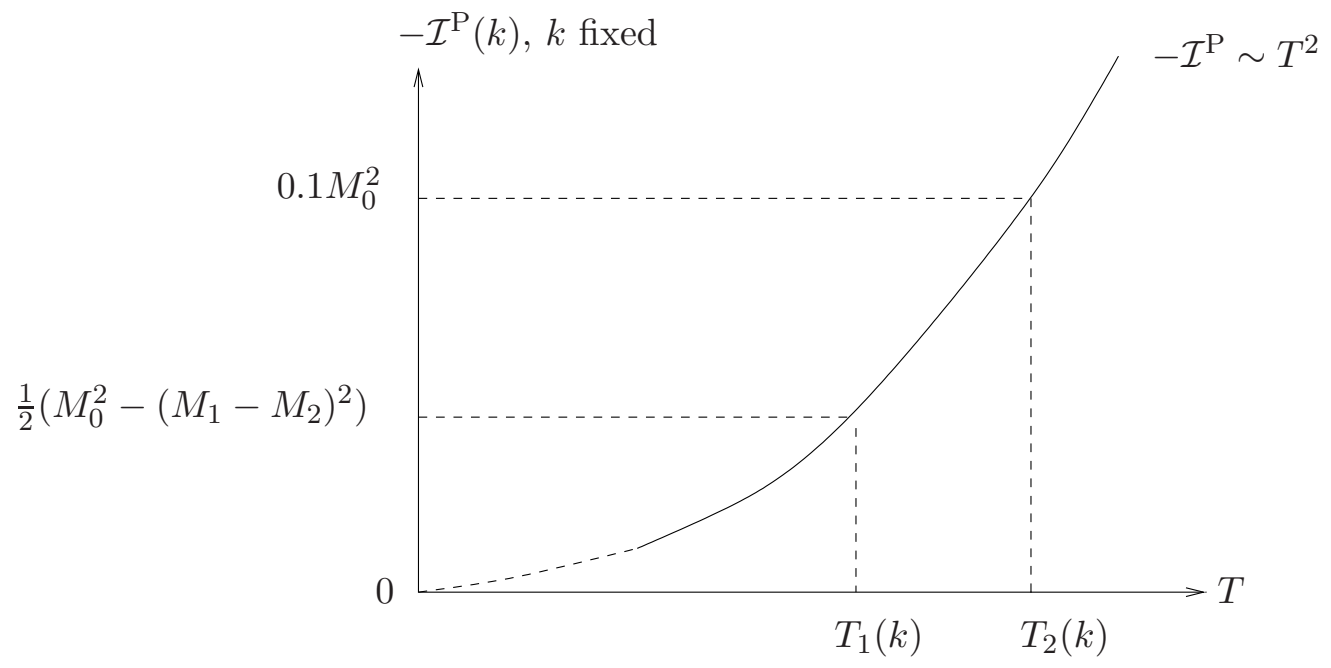

Figure 2. Solving the condition (A.5).

So far the obtained conditions (A.7), (A.6) and (A.12) are common to both case A and case B. Let us now proceed to analyze the conditions (A.3) and (A.4). Define the temperatures $\tilde{T}_{1,2}$ by

$-\left(\mathcal{I}^{\mathrm{P}}+\mathcal{I}^{\mathrm{NP}}\right)\left(k, \tilde{T}_{1}(k)\right)=M_{0}^{2}-\left(M_{1}+M_{2}\right)^{2}$ and $-\left(\mathcal{I}^{\mathrm{P}}+\mathcal{I}^{\mathrm{NP}}\right)\left(k, \tilde{T}_{2}(k)\right)=M_{0}^{2}-\left(M_{1}-M_{2}\right)^{2}$,

then for a mode with momentum $k$ in a thermal bath with temperature $T$,

$$
\begin{array}{cc}
\text { case A occurs if } & \tilde{T}_{1}(k)<T<\tilde{T}_{2}(k), \\
\text { case B occurs if } & T<\tilde{T}_{1}(k) .
\end{array}
$$

Now since $-\left(\mathcal{I}^{\mathrm{P}}+\mathcal{I}^{\mathrm{NP}}\right)<-2 \mathcal{I}^{\mathrm{P}}$, it follows that $\tilde{T}_{2}(k)>T_{1}(k)$ for all $k$. This is important since it implies that the condition (A.12) has always a non-trivial intersection with the conditions (A.15) or (A.16). For each mode with a fixed momentum $k$, depending on the relative sizes of $T_{1}(k), T_{2}(k), \tilde{T}_{1}(k), \tilde{T}_{2}(k)$, either case A or case B or both cases can occur. Specifically we obtain the following result:

When $\tilde{T}_{2}(k)<T_{2}(k)$ :

$$
\begin{aligned}
& \text { if } \tilde{T}_{1}(k)<T_{1}(k), \text { then } \quad \text { case A occurs for } T_{1}(k)<T<\tilde{T}_{2}(k) ; \\
& \text { if } \tilde{T}_{1}(k)>T_{1}(k), \text { then } \quad \text { case A occurs for } \tilde{T}_{1}(k)<T<\tilde{T}_{2}(k) ; \\
& \quad \text { case B occurs for } T_{1}(k)<T<\tilde{T}_{1}(k) .
\end{aligned}
$$

When $\tilde{T}_{2}(k)>T_{2}(k)$ :

$$
\begin{aligned}
\text { if } \tilde{T}_{1}(k)>T_{2}(k), \text { then } & \text { case B occurs for } T_{1}(k)<T<T_{2}(k) ; \\
\text { if } T_{1}(k)<\tilde{T}_{1}(k)<T_{2}(k), \text { then } & \text { case A occurs for } \tilde{T}_{1}(k)<T<T_{2}(k) ; \\
& \text { case B occurs for } T_{1}(k)<T<\tilde{T}_{1}(k) ; \\
\text { if } \tilde{T}_{1}(k)<T_{1}(k), \text { then } \quad & \text { case A occurs for } T_{1}(k)<T<T_{2}(k) .
\end{aligned}
$$


This means that given the temperature $T$ of the thermal bath, modes with different (spatial) momentum $k$ can behave quite differently in regard to the thermalization or stability properties. In particular only a certain window of momentum modes as specified by the inequalities in (A.17)- (A.23) above will be affected for a given $T$. Since the characteristic temperatures $T_{1,2}(k), \tilde{T}_{1,2}(k)$ are monotonic decreasing function of $k$, it means for low temperature $T$, the affected window is in the higher $k$ regime; while for high temperature $T$, the affected window is in the lower $k$ regime. This momentum dependent alternation of the stability or thermalizability is novel and is a combined effect of finite temperature and noncommutative geometry.

Open Access. This article is distributed under the terms of the Creative Commons Attribution Noncommercial License which permits any noncommercial use, distribution, and reproduction in any medium, provided the original author(s) and source are credited.

\section{References}

[1] A. Connes, Noncommutative Geometry, Academic Press, SanDiego U.S.A. (1994) [ISBN:0-12-185860-X].

[2] C.-S. Chu and P.-M. Ho, Noncommutative open string and D-brane, Nucl. Phys. B 550 (1999) 151 [hep-th/9812219] [SPIRES].

[3] C.-S. Chu and P.-M. Ho, Constrained quantization of open string in background B field and noncommutative D-brane, Nucl. Phys. B 568 (2000) 447 [hep-th/9906192] [SPIRES].

[4] V. Schomerus, D-branes and deformation quantization, JHEP 06 (1999) 030 [hep-th/9903205] [SPIRES].

[5] N. Seiberg and E. Witten, String theory and noncommutative geometry, JHEP 09 (1999) 032 [hep-th/9908142] [SPIRES].

[6] S.M. Carroll, J.A. Harvey, V.A. Kostelecky, C.D. Lane and T. Okamoto, Noncommutative field theory and Lorentz violation, Phys. Rev. Lett. 87 (2001) 141601 [hep-th/0105082] [SPIRES].

[7] L. Álvarez-Gaumé and M.A. Vazquez-Mozo, General properties of noncommutative field theories, Nucl. Phys. B 668 (2003) 293 [hep-th/0305093] [SPIRES].

[8] C.-S. Chu, K. Furuta and T. Inami, Locality, causality and noncommutative geometry, Int. J. Mod. Phys. A 21 (2006) 67 [hep-th/0502012] [SPIRES].

[9] S. Minwalla, M. Van Raamsdonk and N. Seiberg, Noncommutative perturbative dynamics, JHEP 02 (2000) 020 [hep-th/9912072] [SPIRES].

[10] M. Van Raamsdonk, The meaning of infrared singularities in noncommutative gauge theories, JHEP 11 (2001) 006 [hep-th/0110093] [SPIRES].

[11] A. Matusis, L. Susskind and N. Toumbas, The IR/UV connection in the non-commutative gauge theories, JHEP 12 (2000) 002 [hep-th/0002075] [SPIRES].

[12] M.M. Sheikh-Jabbari, Discrete symmetries $(C, P, T)$ in noncommutative field theories, Phys. Rev. Lett. 84 (2000) 5265 [hep-th/0001167] [SPIRES].

[13] P. Aschieri, B. Jurčo, P. Schupp and J. Wess, Non-commutative GUTs, standard model and C, P, T, Nucl. Phys. B 651 (2003) 45 [hep-th/0205214] [SPIRES]. 
[14] M. Chaichian, K. Nishijima and A. Tureanu, Spin-statistics and CPT theorems in noncommutative field theory, Phys. Lett. B 568 (2003) 146 [hep-th/0209008] [SPIRES].

[15] J. Gomis and T. Mehen, Space-time noncommutative field theories and unitarity, Nucl. Phys. B 591 (2000) 265 [hep-th/0005129] [SPIRES].

[16] L. Álvarez-Gaumé, J.L.F. Barbon and R. Zwicky, Remarks on time-space noncommutative field theories, JHEP 05 (2001) 057 [hep-th/0103069] [SPIRES].

[17] C.-S. Chu, J. Lukierski and W.J. Zakrzewski, Hermitian analyticity, IR/UV mixing and unitarity of noncommutative field theories, Nucl. Phys. B 632 (2002) 219 [hep-th/0201144] [SPIRES].

[18] E.W. Kolb and M.S. Turner, The Early Universe, Westview Press, Boulder U.S.A. (1990).

[19] J. Bernstein Kinetic theory in the expanding Universe, Cambridge University Press, Cambridge U.K.(1988).

[20] S. Dodelson, Modern Cosmology, Academic Press, San Diego U.S.A. (2003).

[21] J.S. Schwinger, Brownian motion of a quantum oscillator, J. Math. Phys. 2 (1961) 407.

[22] K.T. Mahanthappa, Multiple production of photons in quantum electrodynamics, Phys. Rev. 126 (1962) 329.

[23] P.M. Bakshi and K.T. Mahanthappa, Expectation value formalism in quantum field theory. 2, J. Math. Phys. 4 (1963) 12 [SPIRES].

[24] L.V. Keldysh, Diagram technique for nonequilibrium processes, Zh. Eksp. Teor. Fiz. 47 (1964) 1515 [Soviet Phys. JETP 20 (1965) 1018] [SPIRES].

[25] G. Arcioni and M.A. Vazquez-Mozo, Thermal effects in perturbative noncommutative gauge theories, JHEP 01 (2000) 028 [hep-th/9912140] [SPIRES].

[26] G. Arcioni, J.L.F. Barbon, J. Gomis and M.A. Vazquez-Mozo, On the stringy nature of winding modes in noncommutative thermal field theories, JHEP 06 (2000) 038 [hep-th/0004080] [SPIRES].

[27] W. Fischler et al., Evidence for winding states in noncommutative quantum field theory, JHEP 05 (2000) 024 [hep-th/0002067] [SPIRES].

[28] W. Fischler et al., The interplay between Theta and T, JHEP 06 (2000) 032 [hep-th/0003216] [SPIRES].

[29] K. Landsteiner, E. Lopez and M.H.G. Tytgat, Excitations in hot non-commutative theories, JHEP 09 (2000) 027 [hep-th/0006210] [SPIRES].

[30] K. Landsteiner, E. Lopez and M.H.G. Tytgat, Instability of non-commutative SYM theories at finite temperature, JHEP 06 (2001) 055 [hep-th/0104133] [SPIRES].

[31] F.T. Brandt, J. Frenkel and D.G.C. McKeon, Hard thermal effects in noncommutative U(N) Yang-Mills theory, Phys. Rev. D 65 (2002) 125029 [hep-th/0202202] [SPIRES].

[32] F.T. Brandt, A.K. Das, J. Frenkel, D.G.C. McKeon and J.C. Taylor, Transport equation and hard thermal loops in noncommutative Yang-Mills theory, Phys. Rev. D 66 (2002) 045011 [hep-th/0204192] [SPIRES].

[33] D. Boyanovsky, K. Davey and C.M. Ho, Particle abundance in a thermal plasma: Quantum kinetics vs. Boltzmann equation, Phys. Rev. D 71 (2005) 023523 [hep-ph/0411042] [SPIRES]. 
[34] H.A. Weldon, Simple Rules For Discontinuities In Finite Temperature Field Theory, Phys. Rev. D 28 (1983) 2007.

[35] J.I. Kapusta, Finite temperature field theory, Cambridge University Press, Cambridge U.K. (1989).

[36] M. Le Bellac, Thermal Field Theory, Cambridge University Press, Cambridge U.K. (1996).

[37] J. Gomis, T. Mehen and M.B. Wise, Quantum field theories with compact noncommutative extra dimensions, JHEP 08 (2000) 029 [hep-th/0006160] [SPIRES].

[38] S. Abel, C.-S. Chu and M. Goodsell, Noncommutativity from the string perspective: Modification of gravity at a mm without mm sized extra dimensions, JHEP 11 (2006) 058 [hep-th/0606248] [SPIRES].

[39] C.-S. Chu, B.R. Greene and G. Shiu, Remarks on inflation and noncommutative geometry, Mod. Phys. Lett. A 16 (2001) 2231 [hep-th/0011241] [SPIRES].

[40] F. Lizzi, G. Mangano, G. Miele and M. Peloso, Cosmological perturbations and short distance physics from noncommutative geometry, JHEP 06 (2002) 049 [hep-th/0203099] [SPIRES].

[41] R. Brandenberger and P.-M. Ho, Noncommutative spacetime, stringy spacetime uncertainty principle and density fluctuations, Phys. Rev. D 66 (2002) 023517 [AAPPS Bull. 12N1 (2002) 10] [hep-th/0203119] [SPIRES]. 